\begin{tabular}{|c|c|c|c|c|c|c|}
\hline \multirow{4}{*}{ Impact Factor: } & ISRA (India) & $=3.117$ & SIS (USA) & $=0.912$ & ICV (Poland) & $=6.630$ \\
\hline & ISI (Dubai, UAE & $=0.829$ & РИНЦ (Russia) & $=0.156$ & PIF (India) & $=1.940$ \\
\hline & GIF (Australia) & $=0.564$ & ESJI $(\mathrm{KZ})$ & $=8.716$ & IBI (India) & $=4.260$ \\
\hline & JIF & $=1.500$ & SJIF (Morocco) & $=5.667$ & OAJI (USA) & $=0.350$ \\
\hline
\end{tabular}

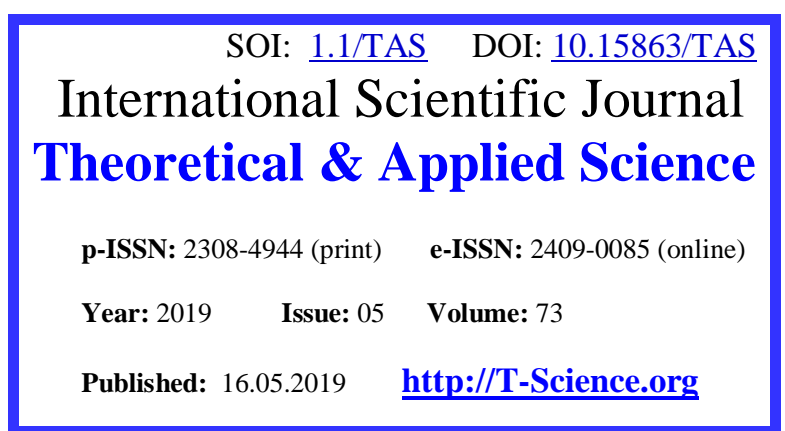

SECTION 7. Mechanics and machine construction
QR - Issue

QR - Article
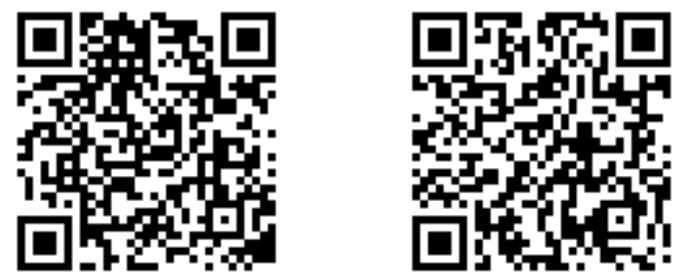

Ismoil Ibragimovich Safarov

Doctor of Physical and Mathematical Sciences, Professor to department of Advanced Mathematics, Tashkent Institute of Chemistry and Technology, Uzbekistan, Safarov54@mail.ru

Nurillo Raximovich Kulmuratov

Senior Lecturer to Department of Technology Engineering, Navoi State Mining Institute, Uzbekistan, nurillo.Kulmuratov.64@mail.ru

\title{
INFLUENCE OF NON-STATIONARY WAVES ON CYLINDRICAL BODY
}

Abstract: In this paper, we study the case of the interaction of a plane compression pulse with a layered cylindrical body in an infinite homogeneous and isotropic elastic medium. The problem is solved by the method of integral Fourier transform. The inverse transformation is carried out numerically by the Romberg method. With a time of toast and a decrease in momentum, the accuracy is at least $2 \%$. Numerical results are obtained taking into account the diffracted waves.

Key words: compression pulse, Fourier transforms Romberg method, Heaviside function, reflection, cylinder, diffraction.

\section{Language: Russian}

Citation: Safarov, I. I., \& Kulmuratov, N. R. (2019). Influence of non-stationary waves on cylindrical body. ISJ Theoretical \& Applied Science, 05 (73), 77-85.

Soi: http://s-o-i.org/1.1/TAS-05-73-15 Doi: crostef https://dx.doi.org/10.15863/TAS.2019.05.73.15

\section{ВЛИЯНИЕ НЕСТАЦИОНАРНЫХ ВОЛН НА ЦИЛИНДРИЧЕСКОЕ ТЕЛО}

Аннотация: В работе исследуется случай взаимодействия плоского импульса сжатия со слоистым цилиндрическим телом в бесконечной однородной и изотропной упругой среде. Поставленная задача решается методом интегрального преобразования Фурье. Обратное преобразование осуществляется численно, методом Ромберга. При времени тоста и уменьшении импульса, точность не менее 2-\%. Численные результаты получены с учетом дифрагированных волн.

Ключевые слова: импульс сжатия, преобразования Фурье, метод Ромберга, функиия Хэвисайда, отражения, ичилиндр, дифракциия.

\section{Введение.}

Развитие системы подземных коммуникаций в сейсмически активных районах потребовало проведения широких исследований [1-4] в этой области. К основным подземным сооружениям относятся системы водоснабжения и канализации, тоннели, подземные переходы, подземные выемки и подводные тоннели. В настоящее время хорошо разработанными являются методики статического и динамического расчета одиночно уложенной трубы, которая без всякого изменения применяются проектными организациями и для расчета труб. В нормативных документах для наземных сооружений рекомендуется применить линейно-спектральную теорию, точнее квазистатическую теорию сейсмостойкости сооружений. Наряду с линейно-спектральной теорией развивается и другая разновидность динамического метода расчета по аналоговым акселограммам, учитывающих особенности площадки строительства. Применительно к подземным сооружениям исследования, связанные с расчетом по акселограммам, развивались по двум основным направлениям, в основу которых положены разные схематизации как самих сооружении, так и взаимодействий их с окружающей средой $[1,2]$. Поведение подземных сооружений при воздействии сейсмовзрывных 


\begin{tabular}{|c|c|c|c|c|c|c|}
\hline \multirow{4}{*}{ Impact Factor: } & ISRA (India) & $=3.117$ & SIS (USA) & $=0.912$ & ICV (Poland) & $=6.630$ \\
\hline & ISI (Dubai, UAE & $=0.829$ & РИНЦ (Russia & $=0.156$ & PIF (India) & $=1.940$ \\
\hline & GIF (Australia) & $=0.564$ & ESJI (KZ) & $=8.716$ & IBI (India) & $=4.260$ \\
\hline & JIF & $=1.500$ & SJIF (Morocce & $=5.667$ & OAJI (USA) & $=0.350$ \\
\hline
\end{tabular}

волн в натурных условиях исследовано в работах $[3,4]$. Проведен ряд сложнейших, дорогостоящих экспериментов в натурных условиях, достоверность результатов которых в настоящее время проверить (повторяемость, идентичность эксперимента) почти невозможно. Экспериментальными исследованиями установлено, что для подземных сооружений основным видом сейсмической нагрузки являются не инерционные силы от веса обделки и горного давления, а динамические изменения сейсмикнапряженного состояния массива при прохождении волн напряжений. При этом имеют место сложные дифракционные волны напряжений - явления, которые в принципе не могут быть учтены изложенными выше теориями сейсмостойкости подземных сооружений. Волновые эффекты, отражения, дифракции и интерференции оказывают существенное влияние на сложное напряженное состояние массива, возникающее в случае нескольких близко расположенных между собой подземных сооружений и расположенных вблизи свободной поверхности. Это и другие волновые эффекты могут быть учтены лишь методами волновой динамики $[5,6]$.

Постановка и методы решения задачи взаимодействия сейсмических волн цилиндрической телом с жидкостью. Ниже решаются задачи взаимодействия нестационарных волн в цилиндрических телах, находящихся в упругой среде.

Пусть в безграничной упругой среде расположен упругий $N$ - слоеный цилиндр, содержащий заполнитель [7] (рис.1). На цилиндр падают нестационарные волны напряжения $\sigma_{x x}^{(i)}$ и $\sigma_{x y}^{(i)}$ цилиндра. Требуется определить динамическое напряженно-деформированное состояние цилиндра и окружающей его среды, вызванное падающим импульсом напряжения.

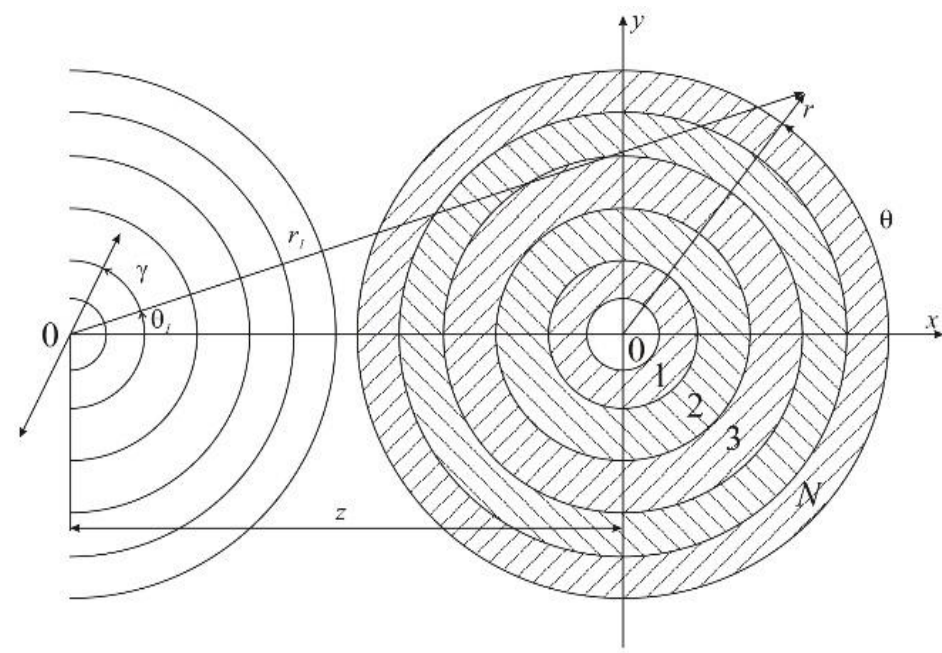

Рис. 1. Действие нестационарных волн на слоистое тело.

Основные уравнения теории упругости для этой задачи о плоской деформации в потенциалах перемещений сводятся к следующим:

$$
\begin{aligned}
& \nabla^{2} \varphi_{j}=\frac{1}{c_{p_{j}}{ }^{2}} \frac{\partial^{2} \varphi_{j}{ }^{\kappa}}{\partial t^{2}} ; \quad(j=1,2 \ldots N) \\
& \nabla^{2} \psi_{j}=\frac{1}{c_{\rho_{j}}{ }^{2}} \frac{\partial^{2} \psi_{j}}{\partial t^{2}},
\end{aligned}
$$

где $\varphi_{j}$ и $\psi_{j}$ являются потенциалами перемещения $j$ го слоя, $c_{p j}$ и $c_{\beta j^{-}}$фазовые скорости распространения волн растяжения и сдвига $j$ - го слоя.

Предположим, что время $t$ отсчитывается с момента, когда падающий импульс коснется поверхности внешнего $(N-1)$-го цилиндра в точке
$r=r_{N}, \theta=0$. До этого момента сохраняется покой. В соответствии с изложенным, задача отыскания поля дифрагированных волн и напряженно-деформированного состояния, вызванного падающим импульсом [8]

$$
\begin{aligned}
& \sigma_{x x}^{(i)}=\sigma_{0} H(\hat{t}), \\
& \sigma_{x y}^{(i)}=\sigma_{0} \frac{v_{N}}{1-v_{N}} H(\hat{t}), \hat{t}=t-\left(x+r_{N}\right) / C_{P N},
\end{aligned}
$$

$\sigma_{0}$ - амплитуда падающих волн; $H(\hat{t})$ - единичная функция Хэвисайда, сводится к решению дифференциальных уравнений (1). Граничные условия на контакте двух цилиндрических поверхностей 


\begin{tabular}{llllll} 
& ISRA (India) $=\mathbf{3 . 1 1 7}$ & SIS (USA) $=\mathbf{0 . 9 1 2}$ & ICV (Poland) & $\mathbf{= 6 . 6 3 0}$ \\
Impact Factor: & ISI (Dubai, UAE) $=\mathbf{0 . 8 2 9}$ & PUHЦ (Russia) $=\mathbf{0 . 1 5 6}$ & PIF (India) & $=\mathbf{1 . 9 4 0}$ \\
& GIF (Australia) $=\mathbf{0 . 5 6 4}$ & ESJI (KZ) & $\mathbf{8 . 7 1 6}$ & IBI (India) & $=\mathbf{4 . 2 6 0}$ \\
& JIF & $=\mathbf{1 . 5 0 0}$ & SJIF (Morocco) $=\mathbf{5 . 6 6 7}$ & OAJI (USA) & $\mathbf{0 . 3 5 0}$ \\
\hline
\end{tabular}

$$
\begin{gathered}
r=a_{\kappa}: \sigma_{r r \kappa}=\sigma_{r r(\kappa+1)} ; \sigma_{r \theta \kappa}=\sigma_{r \theta(\kappa+1)} ; \sigma_{r z \kappa}=\sigma_{r z(\kappa+1)} ; \\
u_{\kappa}=u_{\kappa+1} ; \vartheta_{\kappa}=\vartheta_{\kappa+1} ; w_{\kappa}=w_{\kappa+1} .
\end{gathered}
$$

На бесконечности $(r \rightarrow \infty)$ выполняется условие Зоммерфельда [9]

$\lim _{r \rightarrow \infty} \varphi_{N+1}=0, \quad \lim _{r \rightarrow \infty}\left(\sqrt{r}^{\kappa}{ }^{\kappa}\left(\frac{\partial \varphi_{N+1}}{\partial r}+i \alpha_{N+1} \varphi_{N+1}\right)=0\right.$,

$\lim _{r \rightarrow \infty} \psi_{N+1}=0$,

$\lim _{r \rightarrow \infty}\left(\sqrt{r}^{\kappa}\left(\frac{\partial \psi_{N+1}}{\partial r}+i \beta_{N+1} \psi_{N+1}\right)=0\right.$

Задача решается при следующих начальных условиях [8]:

$$
\begin{gathered}
\frac{\partial \varphi_{j}}{\partial r}+\left.\frac{1}{r} \frac{\partial \psi_{j}}{\partial \theta}\right|_{t=0}=\left.\frac{\partial}{\partial t}\left(\frac{\partial \varphi_{j}}{\partial r}+\frac{1}{r} \frac{\partial \psi_{j}}{\partial \theta}\right)\right|_{t=0}=0 \\
\frac{1}{r} \frac{\partial \psi_{j}}{\partial \theta}-\frac{\partial \varphi_{j}}{\partial r}=\left.\frac{\partial}{\partial t}\left(\frac{1}{r} \frac{\partial \psi_{j}}{\partial \theta}-\frac{\partial \varphi_{j}}{\partial r}\right)\right|_{t=0}=0
\end{gathered}
$$

где $j=1,2, \ldots N$; $N$-число цилиндрических слоев; $j=N$ - окружающая среда.

Интегральное преобразование Фурье. Поле напряжений, вызванных усилиями (2), удовлетворяет волновому уравнению (1), т.е. ему удовлетворяет каждый цилиндрический слой. Для решения сформулированной выше задачи применим $t$-интегральное преобразование Фурье по времени.

$$
\varphi(\xi)=\int_{-\infty}^{+\infty} \varphi(\Omega) e^{-i \xi \Omega} d \Omega ; \quad \varphi(\Omega)=\frac{1}{2 \pi} \int_{-\infty}^{+\infty} \varphi(\xi) e^{i \xi \Omega} d \xi .
$$

Использовав нулевые начальные условия, получим изображенную задачу

$$
\begin{aligned}
& \frac{\partial^{2} \bar{\varphi}_{j}^{F}}{\partial r^{2}}+\frac{1}{r} \frac{\partial \varphi_{j}^{F}}{\partial r}\left(\frac{\Omega^{2}}{C_{P_{j}}^{2}}-\frac{n^{2}}{r^{2}}\right) \bar{\varphi}_{j}^{F}=0, \\
& \frac{\partial^{2} \bar{\psi}_{j}^{F}}{\partial r^{2}}+\frac{1}{r} \frac{\partial \psi_{j}^{F}}{\partial r}\left(\frac{\Omega^{2}}{C_{S_{j}}^{2}}-\frac{n^{2}}{r^{2}}\right) \psi_{j}^{F}=0,
\end{aligned}
$$

где $\Omega$-параметр интегрального преобразования Фурье; $\bar{\varphi}_{j}^{F}, \psi_{j}^{F}$ - изображение преобразования Фурье функций $\varphi_{j}(t)$ и $\psi_{j}(t)$ соответственно. Тогда решение уравнений (4) и (5) будут иметь вид

$$
\left(\frac{\varphi_{j}^{F}(r, \theta, \Omega)}{\psi_{j}^{F}(r, \theta, \Omega)}\right)=\left(\frac{\bar{\varphi}_{j}^{F}(r, \Omega)}{\bar{\psi}_{j}^{F}(r, \Omega)}\right)\left(\frac{\cos \theta}{\sin \theta}\right)
$$

Здесь

$$
\bar{\phi}_{j}^{F}(r, \Omega)=\left\{\begin{array}{c}
A_{n} H_{n}^{(1)}\left(\Omega r / \mathrm{C}_{\mathrm{PN}}\right) \quad \text { при } r \geq \mathrm{r}_{\mathrm{N}}, \\
A_{n j} H_{n}^{(1)}\left(\Omega r / \mathrm{C}_{\mathrm{P}_{\mathrm{j}}}\right)+B_{n j} H^{(2)}\left(\Omega r / \mathrm{C}_{\mathrm{Pj}}\right) \\
\text { при } r_{0} \leq 2 \leq r N(j=1,2, \ldots N-1), \\
A_{n 0} I_{n}\left(\Omega r / \mathrm{C}_{\mathrm{SN}}\right) \quad \text { при } 0 \leq r \leq r_{0} ;
\end{array}\right.
$$

$$
\psi_{j}^{F}(r, \Omega)=\left\{\begin{array}{c}
C_{n J} H_{n}^{(1)}\left(\Omega r / \mathrm{C}_{\mathrm{Sj}}\right)+L_{n J} H_{n}^{(2)}\left(\Omega r / \mathrm{C}_{\mathrm{Sj}}\right) \\
\text { при } r_{0} \leq r \leq r_{N}, \\
C_{n} H_{n}^{(1)}\left(\Omega r / \mathrm{C}_{\mathrm{SN}}\right) \text { при } r \geq \mathrm{r}_{\mathrm{N}}, \\
C_{n 0} I_{n}\left(\Omega r / \mathrm{C}_{\mathrm{S} 0}\right) \text { при } r_{n} \leq r \leq r .
\end{array}\right.
$$

Коэффициенты $A_{n 0}, A_{n j}, A_{n N}, B_{n j}, C_{n j}, C_{n N}$ определяются из граничных условий (7)-(8), которые поставлены на контакте двух цилиндрических поверхностей. Граничные условия при $r=R_{n}$ с учетом падающих волн (1) примут вид
а) $\sigma_{r r N}^{F}+\sigma_{r r N}^{(i) F}=\sigma_{r r(N-1)}^{F}$,
б) $\sigma_{r \theta N}^{F}+\sigma_{r \theta N}^{(i) F}=\sigma_{r \theta(N-1)}^{F}$,
в) $u_{r N}^{F}+u_{r N}^{(i) F}=u_{r(N-1)}^{F}$,
г) $u_{\theta N}^{F}+u_{\theta N}^{(i) F}=u_{\theta(N-1)}^{F}$,

где

a)

$$
\begin{gathered}
\sigma_{r r N}^{(i) F}(\Omega)=\sigma_{01}^{(P)} \sum_{n=0}^{\infty}(-1)^{n} \in_{n} I_{n}\left(\Omega r / C_{P N}\right) \cos n \theta ; \\
\text { б) } \sigma_{r r N}^{F}(\Omega)=\bar{\sigma}_{r r N}^{F}\left(\cos ^{2} \theta+\epsilon_{N} \sin ^{2} \theta\right) ; \\
\text { в) } \sigma_{r \theta N}^{F}=-\bar{\sigma}_{r r}^{F}\left[\left(1-E_{N}\right) \mid 2\right] \sin 2 \theta ; \\
\text { г) } u_{r N}^{F}=\bar{u}_{r N}^{F} \cos \theta ; \\
\text { д) } u_{\theta N}^{F}=\bar{u}_{\theta N}^{F} \sin \theta ; \sigma_{01}^{(P)}=\sigma_{0} e^{-N \Omega / C_{P N}} .
\end{gathered}
$$

Подставив (5) и (6) в граничные условия (7) и (8), получим систему комплексных алгебраических уравнений с $(4 j+3)$ неизвестными в виде

$$
[Z]\{g\}=\{P\},
$$

где

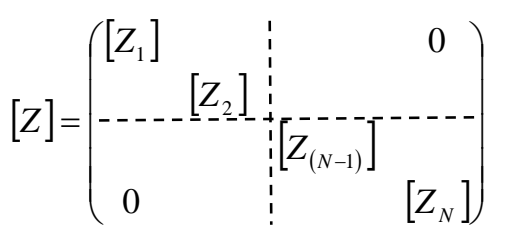

- блочная матрица; $\left\lfloor Z_{j}\right\rfloor$ - матрица размерности $4 \times 4$, элементы которой суть функции Бесселя и Ханкеля $n$-го порядка первого и второго рода; $\{g\}$ - вектор столбца неизвестных коэффициентов; $\{P\}-\left\{0,0 \ldots .0, P_{1 N}, P_{2 N}, P_{3 N}, P_{4 N}\right\}^{T}$.

векторные столбцы, характеризующие падающие нагрузки, где $P_{1 N}, P_{2 N}, P_{3 N}, P_{4 N}$ соответствует $\sigma_{r r N}^{(i) F}, \sigma_{r \theta N}^{(i) F}, u_{r N}^{(i) F}, u_{\theta N}^{(i) F}$.

Пусть ступенчатые волны взаимодействуют с цилиндрическим отверстием при $r=r_{0}$ и 


\begin{tabular}{|c|c|c|c|c|c|c|}
\hline \multirow{4}{*}{ Impact Factor: } & ISRA (India) & $=3.117$ & SIS (USA) & $=0.912$ & ICV (Poland) & $=6.630$ \\
\hline & ISI (Dubai, UAE & $=0.829$ & РИНЦ (Russia) & $=0.156$ & PIF (India) & $=1.940$ \\
\hline & GIF (Australia) & $=0.564$ & ESJI (KZ) & $=8.716$ & IBI (India) & $=4.260$ \\
\hline & JIF & $=1.500$ & SJIF (Morocco) & $=5.667$ & OAJI (USA) & $=0.350$ \\
\hline
\end{tabular}

отверстием свободного от напряжения $\left(\left.\sigma_{r r}\right|_{r=a}=\left.\sigma_{r \theta}\right|_{r=a}=0\right)$

Единственным напряжением, которое не обращается в нуль при $r=r_{0}$, является кольцевое напряжение $\sigma_{\theta \theta n} / \sigma_{0}$. Применив преобразование Фурье к уравнению движения и граничным условиям [5], получим выражение для кольцевых напряжений при

$\sigma_{r r}=\sigma_{0} H(t) \cos n t, \sigma_{r \theta}=\tau_{0} H(t) \sin \theta:$

$\sigma_{\theta \theta n}^{*}=\frac{\sigma_{\theta \theta n}\left(r_{01} \theta, t\right)}{\sigma}=\frac{1}{2 \pi} \int_{-\infty}^{\infty} \frac{\Delta_{1}\left(r_{0} \Omega\right) e^{i \Omega t}}{\Omega_{1}\left[\Delta_{2} \Delta_{3}+\Delta_{4} \Delta_{5}\right]} d \Omega$,

$\Delta_{1}\left(r_{01} \Omega\right)=$

$=\left(\Delta_{3}+\tau_{0} E\right)\left[2 \Omega H_{n-1}^{(1)}(\Omega)-\left(\left(2 n^{2}+2 n\right)+\Omega^{2}\right) H_{n}^{(1)}(\Omega)\right]+$

$+\left[\tau_{0} \Delta_{2}-\Delta_{4}\right] \times$

$\times\left[2 n(n+1) H_{n}^{(1)}\left(\left(C_{P 1} / C_{S 1}\right) \Omega\right)+\frac{2 C_{P} n \Omega}{C_{S 1}} H_{n-1}^{(1)}\left(\frac{C_{P}}{C_{S}} \Omega\right)\right]$.

Выражение $\left.\Delta_{k}(k=1,2,3,4,5)\right)$ приведено в работе [9]. Несобственный интеграл (10) решается численно по разработанным алгоритмам [14]. Практические вычисления (10) на ЭВМ можно провести следующим образом. Поскольку численное интегрирование в бесконечных пределах немыслимо, то интеграл (10) заменяется на

$$
\sigma_{\theta \theta n}^{*}=\frac{1}{2 \pi} \int_{\omega_{a}}^{\omega_{b}} \frac{\Delta_{1}\left(r_{01} \Omega_{1}\right)}{\Omega_{1}\left[\Delta_{2} \Delta_{3}+\Delta_{4} \Delta_{5}\right]} e^{-i \Omega t} d \Omega
$$

Значения пределов интегрирования $\omega_{a}, \omega_{b}$ подбираются в зависимости от вида падающего импульса. Численные значения спектральной плотности $\sigma_{r r}^{(i) F}(\Omega)$ из (9) конечного падающего импульса лишь в небольшом диапазоне частоты $\Omega$ существенно отличается от нуля. Пределы интегрирования $\omega_{a}, \omega_{b}$ следует подбирать в соответствии с этим диапазоном и с учетом требуемой точности. При этом остается открытым вопрос о том, какую погрешность внесет пренебрежение вкладом интегралов типа (10) в пределах интегрирования от $-\infty$ до $\omega_{a}$ и от $\omega_{b}$ до $\infty$.

Численное суммирование бесконечной суммы (10), разумеется, также невозможно. Однако в [10] показано, что при достаточно больших $\mathrm{n}$ (n-порядок функций Бесселя и Ханкеля) можно построить асимптотическое представление общего члена этой суммы. В результате получим либо оценку погрешности перехода от бесконечной суммы к конечной, либо приближенное суммирование бесконечной суммы. Ввиду сказанного сохраним в (10) бесконечную сумму. Расчет по рассматриваемому методу сводится к построению двух алгоритмов вычисления: коэффициентов $Z_{k e}(\Omega)(k, e=1,2)$ (11) и интеграла (10). Первый и второй алгоритмы не зависят от вида математической модели объекта.

Алгоритм вычисления. Величина $\sigma_{\theta \theta n}^{F} / \sigma_{0}$ из (11) вычисляется на ЭВМ следующим образом. Задаются все числовые параметры, необходимые для вычислений. Вводятся следующие обозначения: $\quad x_{1}=\Omega, x_{2}=n_{1} \Omega, \quad$ где $n_{1}=\frac{C_{P 1}}{C_{S 1}} ; \Omega=\frac{\omega \alpha}{C_{P 1}}, x_{2}=n_{1} \Omega$. Для двух значений $x_{k}(k=1,2)$ определяется функция Бесселя $I_{n}(\xi)$ и $N_{n}(\xi),(n=1,2 \ldots 10)$. Указанные массивы рассчитываются по формуле

$u_{n}(\xi)=\frac{2(n-1)}{\xi} u_{n-1}(\xi)-u_{n-2}(\xi), u_{n}(\xi)=I_{n}(\xi), N_{n}(\xi)$

Как показано в [11], абсолютное значение функции Бесселя быстро уменьшается с ростом индекса $n$, начиная с момента, когда индекс превышает аргумент. В этом случае, непосредственное использование формулы (12) не приводит к цели. Тем не менее, расчет по (12) возможен, если по рекуррентной формуле

$$
\bar{I}_{n}(\xi)=\frac{2(n-1)}{\xi} \bar{I}_{n+1}(\xi)-\bar{I}_{n+2}(\xi)
$$
в направлении убывания индекса (от $n=N$ до $n=0$ ) рассчитывается вспомогательная функция $\bar{I}_{n}(\xi)$. Для расчета (13) задаются некоторые начальные значения

$$
\bar{I}_{N_{*}+1}(\xi)=0, \quad \bar{I}_{N_{*}}=\varepsilon_{*}
$$

и начальный индекс $n=N_{*}$, определяемый из уравнения

$$
\frac{(2 \xi)^{N_{*}} N_{*} !}{\left(2 N_{*}+1\right)}=\varepsilon_{*} .
$$

Здесь $\varepsilon_{-}$погрешность расчета, соответствующая требуемой точности. Далее определяется значение функции Бесселя:

$$
I_{n}(\xi)=\bar{I}_{n}(\xi) I_{0}(\xi) / \bar{I}_{0}(\xi),
$$

причем для нахождения $I_{0}(\xi)$

можно использовать зависимость

$$
I_{0}(\xi)=\frac{1}{\pi} \int_{0}^{x} \cos (\xi \cos x) d x
$$

С помощью функций Бесселя и Неймана решается уравнение (16).

Функцию

$$
\chi_{1}\left(r_{0} \Omega_{1}\right)=\left(\Delta_{1}\left(r_{0}, \Omega_{1}\right) / \Omega_{1}\left(\Delta_{2} \Delta_{3}+\Delta_{4} \Delta_{5}\right)\right) e^{i \Omega t}
$$

можно численно интегрировать, записав ее в виде

$$
\chi_{1}\left(r_{0} \Omega_{1}\right)=x_{1}\left(r_{0}, \Omega_{1}\right)-i \mathrm{x}_{1}\left(r_{0}, \Omega_{1}\right) \text {. }
$$




\begin{tabular}{|c|c|c|c|c|c|c|}
\hline \multirow{4}{*}{ Impact Factor: } & ISRA (India) & $=3.117$ & SIS (USA) & $=0.912$ & ICV (Poland) & $=6.630$ \\
\hline & ISI (Dubai, UAI & $=0.829$ & РИНЦ (Russia) & $=0.156$ & PIF (India) & $=1.940$ \\
\hline & GIF (Australia) & $=0.564$ & ESJI (KZ) & $=8.716$ & IBI (India) & $=4.260$ \\
\hline & JIF & $=1.500$ & SJIF (Morocco) & $=5.667$ & OAJI (USA) & $=0.350$ \\
\hline
\end{tabular}

Падающий $\quad$ импульс $\quad \sigma_{x x}^{(i) F}(\Omega)$

описывается выражением

$$
\sigma_{x x}^{(i) F}(\Omega)=f_{1}(\Omega)-i f_{2}(\Omega)
$$

где $f_{1}(\Omega), f_{2}(\Omega)$ _ вещественные функции.

Использовав формулу Эйлера для $\exp (i \Omega t)$, разделив (18) на вещественную и мнимую (19) части, после некоторых преобразований получим в виде:

$$
\sigma_{\theta \theta n}^{F}=\frac{1}{2 \pi} \int_{-\infty}^{\infty}\left[x_{1}(\Omega)-i x_{2}(\Omega)\right] d \Omega
$$

Разделив интеграл (20) на два слагаемых

$\sigma_{\theta \theta n}^{F}=\frac{1}{2 \pi} \int_{-\infty}^{0}\left[x_{1}(\Omega)-i x_{2}(\Omega)\right] d \Omega+\frac{1}{2 \pi} \int_{0}^{\infty}\left[x_{1}(\Omega)-i x_{2}(\Omega)\right] d \Omega$

и заменив в первом интеграле переменную $\Omega$ на $-\Omega$, будем иметь

$\sigma_{\theta \theta n}^{F}=\frac{1}{2 \pi} \int_{0}^{\infty}\left[x_{1}\left(\Omega_{1}\right)-x_{1}\left(-\Omega_{1}\right)\right]-i\left[x_{2}\left(\Omega_{1}\right)-x_{2}\left(-\Omega_{1}\right)\right] d \Omega$.

Поскольку (22) представляет собой обратное преобразование Фурье и в левой части содержит вещественную величину [11], то справедливо соотношение

$$
x_{1}\left(\Omega_{1}\right)=-x_{1}\left(-\Omega_{1}\right) ; x_{2}\left(\Omega_{1}\right)=-x_{2}\left(-\Omega_{1}\right) .
$$

Учитывая его, из (23) окончательно получаем

$$
\begin{gathered}
\sigma_{\theta \theta n}^{F}=\frac{1}{\pi} \int_{0}^{\infty} x_{1}(\Omega) d \Omega, \\
\sigma_{\theta \theta n}^{F}=\frac{1}{\pi} \int_{\omega_{a}}^{\omega_{b}} x_{1}(\Omega) d \Omega .
\end{gathered}
$$

Величину интеграла (24) найдем численно с помощью метода Ромберга [3,4]. Основной алгоритм этого метода приведен в первой главе. При вычислении интеграла по методу Ромберга приходится многократно вычислять подынтегральную функцию. Обратное преобразование Фурье для некоторого изображения, оригинал которого заранее известен, показало, что при длине шага интегрирования 0,01 погрешность процедуры не превышает 0,3-0,5\%.

Представлены численные результаты для кольцевого напряжения при $r=r_{0}$, вызванного набегающей плоской ударной волной со ступенчатым распределением напряжения во времени. Получены численные результаты при $v=0,25 ; \quad \mathrm{C}_{\mathrm{S} 1} / \mathrm{C}_{\mathrm{P} 1}=0,5 ; \theta=0^{0} u 90^{\circ} . \quad$ Для определения интеграла (25) границы интеграла $\omega_{a}$ и $\omega_{b}$ выбрали $\left\lfloor 10^{-4}-N\right\rfloor, N=1,2 \ldots 5$, а шаг $h=0,1 ; 0,01 ; 0,001$. При $N=5$ и $N=6$ значение кольцевого напряжения отличается от предыдущего пятым знаком после запятой (рис.2). Изменение $\sigma_{\theta \theta}^{*}$ в зависимости от $\tau$ при различных $n=0,1,2,3,4,5$ показано на рис.3-4. Результаты наших численных расчетов сравнивали с известными результатами [9]. Полученные значения отличаются приблизительно на $30 \%$ при $n=0,1$ : максимальное кольцевое напряжение при $h=0,01$ и $\theta=90$ составляет 2,962/3,0; а по работе [11,12] - 3,28/3,0 $(\tau \approx 4,71)$.

Дифракции нестационарных волн на цилиндрическом теле. Пусть внутренняя граница $\left(r=r_{0}\right)$ свободна от напряжения, а на контакте c окружающей средой выполняется условие равенства перемещений и напряжений (7) [11]. После преобразования Фурье получим цилиндрические уравнения Бесселя (13) и (16), решение которых имеет вид (7) и (8). В нашей задаче будет шесть произвольных постоянных, которые определяются из граничных условий (8). Вот некоторые из них:

$$
\left.\begin{array}{c}
\sigma_{r r_{2}}=2 \mu_{2} r^{-2} \sum_{k=1}^{2} \sum_{n=0}^{\infty} \int_{-\infty}^{+\infty}\left[C_{n k} \varepsilon_{1 n}^{(k)}+D_{n k} \varepsilon_{2 n}^{(k)}\right] e^{i \Omega \tau} d \Omega, \\
\sigma_{\theta \theta_{2}}=2 \mu_{2} r^{-2} \sum_{k=1}^{2} \sum_{n=0}^{\infty} \int_{-\infty}^{+\infty}\left[C_{n k} \varepsilon_{3 n}^{(k)}+D_{n k} \varepsilon_{4 n}^{(k)}\right] e^{i \Omega \tau} d \Omega, \\
\sigma_{\mathrm{r} \theta_{2}}=2 \mu_{2} r^{-2} \sum_{k=1}^{2} \sum_{n=0}^{\infty} \int_{-\infty}^{+\infty}\left[C_{n k} \varepsilon_{5 n}^{(k)}+D_{n k} \varepsilon_{6 n}^{(k)}\right] e^{i \Omega \tau} d \Omega, \\
\sigma_{r r_{1}}=2 \mu_{1} r^{-2} \sum_{k=1}^{2} \sum_{n=1}^{\infty} \int_{-\infty}^{+\infty}\left[A_{n} \delta_{n}^{(1)}+B_{n} \delta_{n}^{(2)}\right] e^{i \Omega \tau} d \Omega,
\end{array}\right\}
$$

где $C_{n k}, D_{n k}, A_{n}, B_{n}$ - произвольные постоянные: $C_{n k}=\delta_{k n}^{(c)} / \Delta_{n}, \quad D_{n k}=\delta_{k n}^{(D)} / \Delta_{n}, \quad A_{n}=\delta_{n}^{(A)} / \Delta_{n}$, $B_{n}=\delta_{n}^{(B)} / \Delta_{n} ; \quad \delta_{k n}^{(k)} \quad$ и $\quad \Delta_{n_{-}} \quad$ квадратные комплексные матрицы (6х6). Остальные элементы тензора напряжений записываются аналогично (26).

$$
\begin{aligned}
& C_{n k}=\operatorname{Re} C_{n k}+i \operatorname{Im} C_{n k}, D_{n k}=\operatorname{Re} D_{n k}+i \operatorname{Im} D_{n k}, \\
& A_{n}=\operatorname{Re} A_{n}+i \operatorname{Im} A_{n}, B_{n}=\operatorname{Re} B_{n}+i \operatorname{Im} B_{n}, \\
& \delta_{n}^{(e)}=\operatorname{Re} \delta_{n}^{(e)}+i \operatorname{Im} \delta_{n}^{(e)}, e=1,2, \\
& \varepsilon_{m n}^{(k)}=\operatorname{Re} \varepsilon_{m n}^{(k)}+i \operatorname{Im} \varepsilon_{m n}^{(k)}, \\
& e^{i \Omega t}=\cos \Omega \mathrm{t}+\mathrm{i} \sin \Omega \mathrm{t}, m=1,2, \ldots ., 5,
\end{aligned}
$$

Подставив (27) в (26), после некоторых преобразований получим тензор напряжений

$$
\sigma_{j i}=\sum_{k=1}^{2} \sum_{n=0}^{\infty} \int_{\omega_{a}}^{\omega_{b}} R e \sigma_{i j}^{\prime} d \Omega
$$

Все указанные процедуры заложены в памят машины. Разработан универсальный алгоритм 


\begin{tabular}{|c|c|c|c|c|c|c|}
\hline \multirow{4}{*}{ Impact Factor: } & ISRA (India) & $=3.117$ & SIS (USA) & $=0.912$ & ICV (Poland) & $=6.630$ \\
\hline & ISI (Dubai, UAE & $=0.829$ & РИНЦ (Russia & $=0.156$ & PIF (India) & $=1.940$ \\
\hline & GIF (Australia) & $=0.564$ & ESJI (KZ) & $=8.716$ & IBI (India) & $=4.260$ \\
\hline & JIF & $=1.500$ & SJIF (Morocce & $=5.667$ & OAJI (USA) & $=0.350$ \\
\hline
\end{tabular}

вычисления интегралов типа (28). Результаты расчетов приведены на рис. 5 при $\theta=90^{\circ}$ $\left(v_{1}=0,2 ; v_{2}=0,25 ; r_{0} / r_{1}=0,5 ; E_{1} / E_{2}=0,1 ; \eta=0,1\right)$

Полученные данные сопоставлены с известными результатами [25,26]. При интегрировании предела $\omega_{a}=10^{-4}, \omega_{b}=4, h=10^{-2}$ результаты моего расчета отличаются от данных на $\approx 20 \%$. Аналогичные результаты получены для цилиндрических оболочек, находящихся в упругой среде. Уравнение движения цилиндрических оболочек имеет вид [12], а окружного напряжения $\sigma_{\theta \theta}^{*}$ в оболочке, но здесь $C_{n 2}=D_{n 2}=0$. Изменение окружного напряжения $\sigma_{\theta \theta}^{*}\left(\theta=90^{0}, r=r_{0}\right)$ в зависимости от $\tau$, как показано на рис.6, где 1-результаты [12], 2- результаты авторов при данных $\left(h / r=0,04 ; h=\left(r_{1}-r_{0}\right) / 2\right)$. Аналогичные результаты получены в работе [12], однако авторы считают, что $\epsilon_{1}=h^{2} / 12 R^{2}=0$, т.е. они учитывают изгибающий момент. $\mathrm{B}$ случае упругих цилиндрических тел определение напряженнодеформированного состояния объекта и окружающей его среды при действии нестационарных волн основано на построении последовательности падающих импульсов из стационарных компонентов, где каждый импульс представляет собой изменение во времени неустановившегося напряжения в падающей волне. На рис.7 показано изменение окружного напряжения

$$
\sigma_{\theta \theta}^{*}\left(\theta=90^{\circ}, r=r_{0}, r=r_{0}+\left(r_{1}-r_{0}\right) / 2, r=r_{1}\right)
$$

в зависимости от $\tau$.

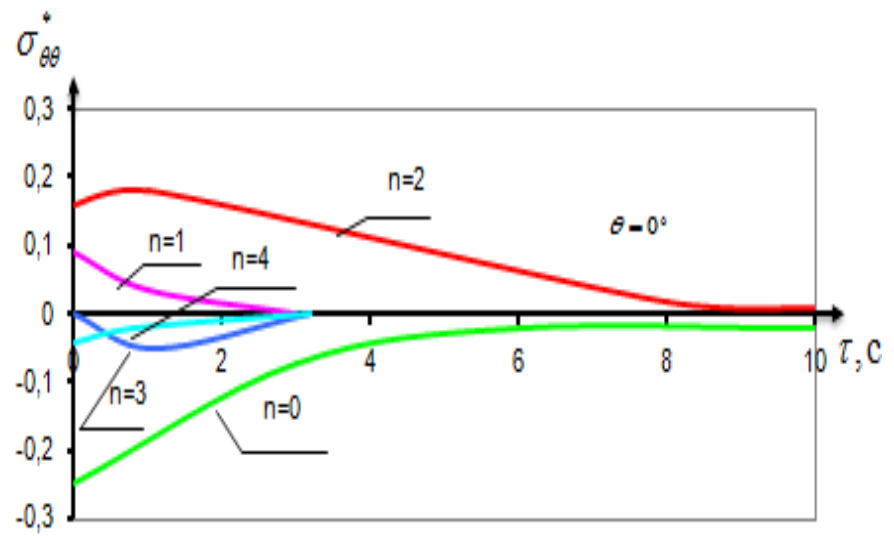

Рис.2. Зависимость кольцевых напряжений от времени, при различных $\boldsymbol{n}$

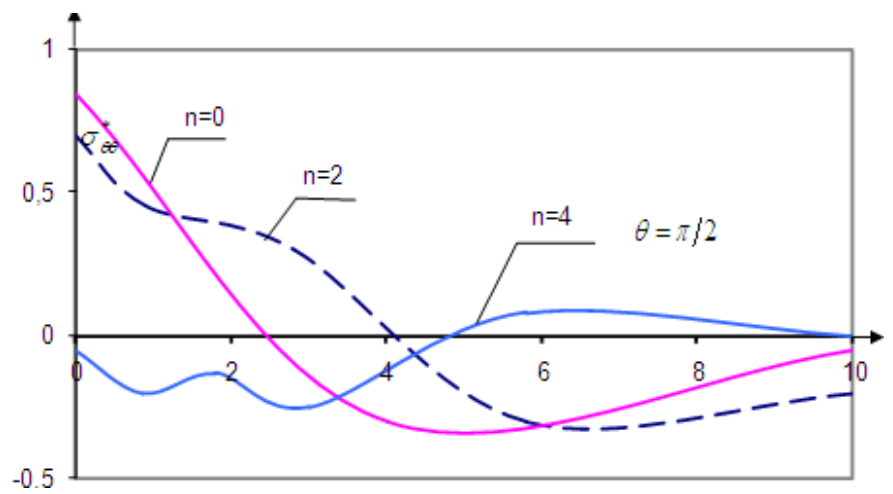

Рис.3. Зависимость кольцевых напряжений от времени, при различных $n$ 


\begin{tabular}{|c|c|c|c|c|c|c|}
\hline \multirow{4}{*}{ Impact Factor: } & ISRA (India) & $=3.117$ & SIS (USA) & $=0.912$ & ICV (Poland) & $=6.630$ \\
\hline & ISI (Dubai, UAE & $=0.829$ & РИНЦ (Russia) & $=0.156$ & PIF (India) & $=1.940$ \\
\hline & GIF (Australia) & $=0.564$ & ESJI (KZ) & $=8.716$ & IBI (India) & $=4.260$ \\
\hline & JIF & $=1.500$ & SJIF (Morocco) & $=5.667$ & OAJI (USA) & $=0.350$ \\
\hline
\end{tabular}

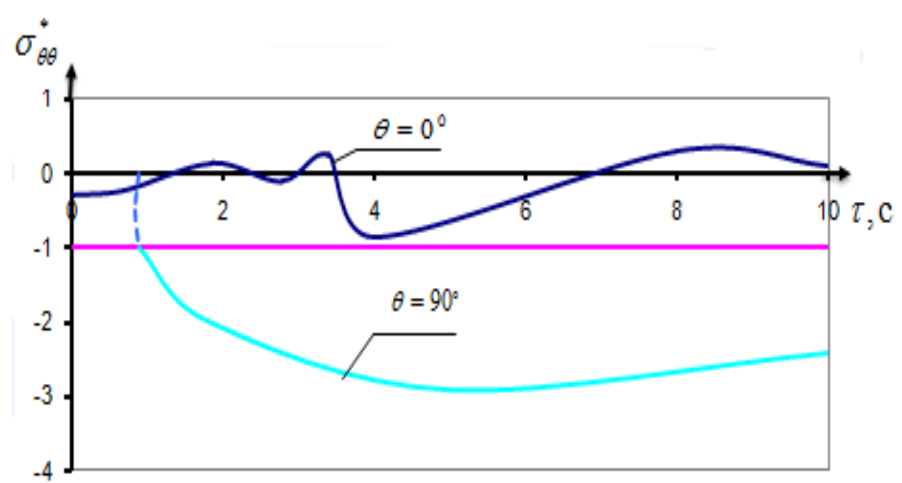

Рис.4. Зависимость кольцевого напряжения от времени

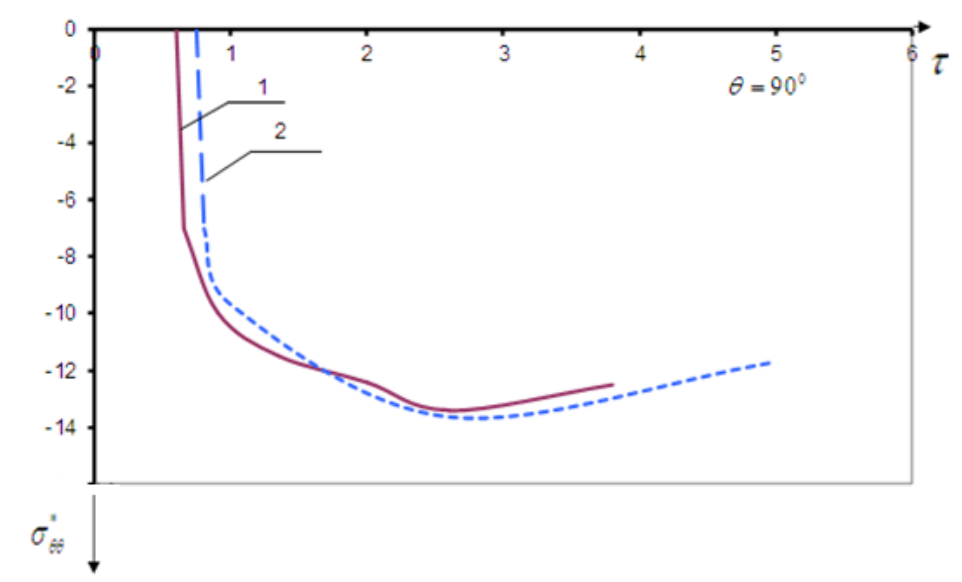

Рис.5. Зависимость кольцевого напряжения поверхности слоя от времени

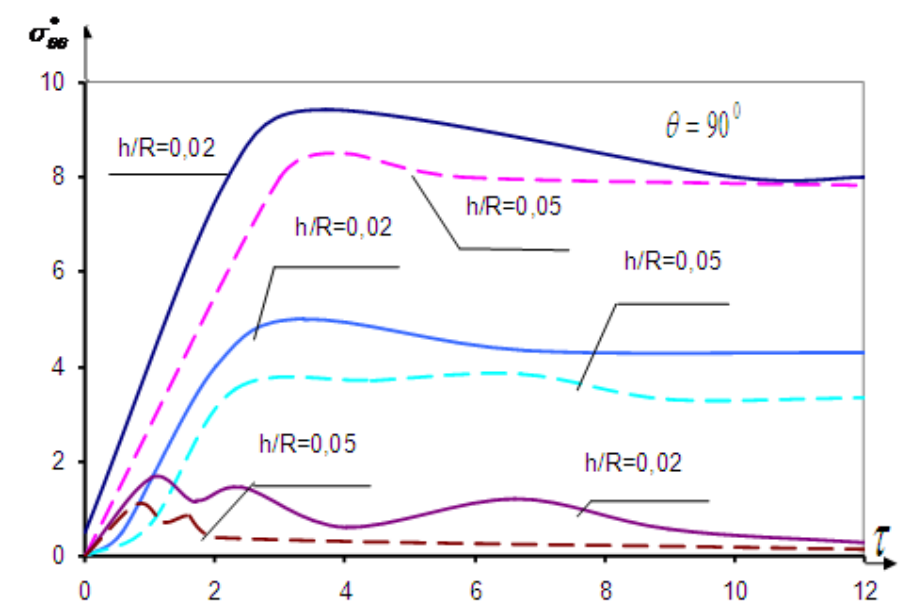

Рис.6. Зависимость безразмерного кольцевого напряжения от при различные $h / R$ 


\begin{tabular}{|c|c|c|c|c|c|c|}
\hline \multirow{4}{*}{ Impact Factor: } & ISRA (India) & $=3.117$ & SIS (USA) & $=0.912$ & ICV (Poland) & $=6.630$ \\
\hline & ISI (Dubai, UAE & $=0.829$ & РИНЦ (Russia & $=0.156$ & PIF (India) & $=1.940$ \\
\hline & GIF (Australia) & $=0.564$ & ESJI (KZ) & $=8.716$ & IBI (India) & $=4.260$ \\
\hline & JIF & $=1.500$ & SJIF (Morocce & $=5.667$ & OAJI (USA) & $=0.350$ \\
\hline
\end{tabular}

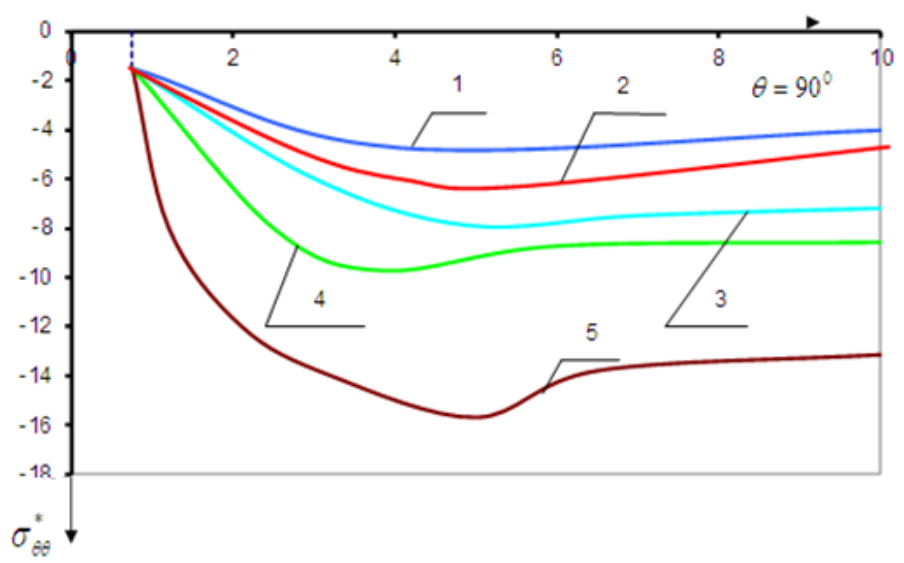

Рис.7. Зависимость кольцевого напряжения внутренней поверхности цилиндрического слоя от времени: 1-гранит-бетон; 2-песчаник-бетон; 3-мягкий грунт-бетон;4-гранит-сталь;5-песчаник-сталь.

Различие между напряжениями на внешней и внутренней поверхностях достигают, $\approx 15-20 \%$, a различие между напряжениями на средней и внутренней поверхностях $\approx 10 \%\left(r_{0} / r_{1}=0,5\right)$. Расчеты показывают, что $\tau=12 \alpha / C_{P_{1}}$ результаты данного исследования приближаются к точному статическому значению $\sigma_{\theta \theta}^{*}=8,13$. Зависимость окружного напряжения от $\tau$ представлена на рис.7. Видно, что максимальное напряжение и перемещения существенно зависят от $\bar{\eta}$ и $E$.

Дифракции упругих нестационарных волн в двухслойном цилиндрическом теле. Пусть на упругое двухслойное цилиндрическое тело при $t>0$ падает нестационарная ступенчатая нагрузка (1). На границах контакта ставится условие жесткого контакта. Тензор напряжения в каждом слое записывается в виде

$\sigma_{i j}^{(k)}\left(r_{1} \theta_{1} t\right)=\frac{1}{\pi} \sum_{n=0}^{\infty} \int_{\omega_{a}}^{\omega_{b}} \operatorname{Re} \sigma_{n i j}^{(k)}\left(r_{1} \theta_{1} \Omega\right) d \Omega, \mathrm{k}=1,2,3$.

Тензор напряжения $\sigma_{i j}^{(k)}$ представляет собой функции Бесселя и Ханкеля первого и второго рода n-го порядка. Интеграл (29) вычисляется по разработанному алгоритму первой главы. При решении ограничивались пятью членами ряда (29), так как удержание следующих членов ряда практически не влияет на результаты. Например, удержание десяти членов (29) изменяет значение напряжения менее, чем на 2-3\%. В расчетах были использованы следующие параметры: $r_{0} / r_{2}=0,2 ; \quad r_{1} / r_{2}=0,6 ; \quad v_{1}=0,2 ;$ $v_{2}=0,25 ; \quad v_{3}=0,2 ; \quad E_{1} / E_{2}=0,3 ; \quad E_{3} / E_{2}=0,1 ;$ $\rho_{1} / \rho_{2}=0,3 ; \rho_{3} / \rho_{2}=0,1$.

\section{Выводы.}

В работе предложена методика и алгоритм решения задачи дифракции упругих волн на многослойном цилиндрическом теле. В случае упругих цилиндрических тел определение напряженно-деформированного состояния объекта и окружающей его среды при действии нестационарных волн основано на построении падающих импульсов из стационарных компонентов, где каждый импульс представляет собой изменение во времени неустановившегося напряжения в падающей волне.

\section{References:}

1. Safarov, I. I., Teshaev, M. X., Akhmedov, M. S., \& Boltaev, Z. I. (2017). Distribution Free Waves in Viscoelastic Wedge with and Arbitrary Angle Tops. Applied Mathematics, 8, pp.736-745.

2. Safarov, I. I., Teshaev, M. K., Boltaev, Z. I., \& Nuriddinov, B. Z. (2017). Of Own and Forced Vibrations of Dissipative Inhomogeneous
Mechanical Systems. Applied Mathematics, 8, pp.1001-1015.

3. Safarov, I. I. (1992). Kolebaniya $i$ volni $v$ dissipativno nedorodnix sredax i konstruksiyax. (p.250). Tashkent: Fan.

4. Safarov, I. I., Akhmedov, M., \& Umarov, A. (2017). Own vibrations of toroidal shell with 


\begin{tabular}{llllll} 
& ISRA (India) $=\mathbf{3 . 1 1 7}$ & SIS (USA) & $=\mathbf{0 . 9 1 2}$ & ICV (Poland) & $=\mathbf{6 . 6 3 0}$ \\
Impact Factor: & ISI (Dubai, UAE) $=\mathbf{0 . 8 2 9}$ & PUHL (Russia) $=\mathbf{0 . 1 5 6}$ & PIF (India) & $=\mathbf{1 . 9 4 0}$ \\
& GIF (Australia) $=\mathbf{0 . 5 6 4}$ & ESJI (KZ) & $=\mathbf{8 . 7 1 6}$ & IBI (India) & $=\mathbf{4 . 2 6 0}$ \\
& JIF & $\mathbf{1 . 5 0 0}$ & SJIF (Morocco) $=\mathbf{5 . 6 6 7}$ & OAJI (USA) & $\mathbf{0 . 3 5 0}$ \\
\hline
\end{tabular}

flowing liquid. (p.177). Lambert Academic Publishing (Germany).

5. Safarov, I. I., Teshaev, M. K., \& Boltaev, Z. I. (2017). Distribution of harmonic waves in expansion plastic and cylindrical viscoelastic bodies. (p.218). Open Science Publishing Raleigh, North Carolina, USA.

6. Rashidov, T. R. (1973). Dinamicheskaya teoriya seysmostoykosti slojnix sistem podzemnix soorujeniy. (p.182). Tashkent: Fan.

7. Mau, M. (1963). Dinamicheskiye napryajeniya i smeshyeniye vblizi silindricheskoy poverxnosti razriva ot ploskoy garmonicheskoy volni sdviga. Prikladnaya mexanika, perevod s angliyskogo, t.30, ser Ye, № 3, pp.117-126.

8. Safarov, I. I., Boltaev, Z. I., Axmedov, M. S., \& Rajabov, O. (2017). Numerical solution of the problem on the impact plane non-stationary elastic waves by a cylindrical body. Discover 2017, 53, (256). April. pp.255-265.

9. Safarov, I. I. (2018). On the Vibration of Parallel Cylindrical Shells Under the Action of Harmonic Waves. International Journal of Emerging Engineering Research and Technology Volume 6, Issue 6, pp.1-8.

10. Safarov, I. I., \& Teshaev, M. K. (2018). Damping of Vibrations of DissipativeInhomogeneous Multiayer Plates and Shells Interacting with the Medium. International Journal of Emerging Engineering Research and Technology Volume 6, Issue 6, pp.33-44. ISSN 2349-4395.

11. Safarov, I. I., Teshaev, M. K., \& Boltaev, Z. I. (2018). Own Vibrations of Bodies Interacting with Unlimited Deformable Environment. Open Access Library Journal. 2018, Volume 5, e4432 Access Library Journal, 5: e4432. pp.1-22.

12. Safarov, I. I., Teshaev, M. K., Akhmedov, M. S. (2018). Free Oscillations of a Toroidal Viscoelastic Shell with a Flowing Liquid. American Journal of Mechanics and Applications, 6(2): 37-49.

13. Safarov, I. I., Teshaev, M. K., \& Akhmedov, M. S. (2018). Free Oscillations of a Toroidal Viscoelastic Shell with a Flowing Liquid. American Journal of Mechanics and Applications, 6(2): 37-49.

14. Safarov, I. I., Teshaev, M. K., Boltaev, Z. I., \& Akhmedov, M. S. (2018). Of Own Vibrations of Cylindrical Bodies in the Deformable MediumSpecific Vibrations of Cylindrical Bodies in the Deformed Environment. World Wide Journal of Multidisciplinary Research and Development (WWJMRD) 4(2): 42-53.

15. Safarov, I. I., Teshayev, M. K., Boltayev, Z. I., \& Nuriddinov, B. Z. (2018). Own Waves in a Cylindrical Shell in Contact with a Viscous Liquid. World Wide Journal of Multidisciplinary Research and Development (WWJMRD) 4(1): 140-151.

16. Safarov, I. I., \& Teshaev, M. K. (2018). Vibration Protection of Mechanical Systems Consisting of Solid and Deformable Bodies. EJERS, European Journal of Engineering Research and Science. Vol. 3, No. 9, September, pp.18-28. 